\title{
Universality class of fiber bundles with strong heterogeneities
}

\author{
R. C. Hidalgo ${ }^{1}$, K. Kovács ${ }^{2,3}$, I. Pagonabarraga ${ }^{4}$ and F. Kun ${ }^{2}$
}

AMADE, Departament de Fúsica and Departament de Enginyeria Mecànica de la Construcció Industrial Universitat de Girona Ave. Montilivi s/n, 17071-Girona, Spain

2 Department of Theoretical Physics, University of Debrecen, P.O.Box: 5, H-4010 Debrecen, Hungary

3 Department of Appl. Math. and Prob. Theory, University of Debrecen, P.O.Box: 12, H-4010 Debrecen, Hungary

4. Departament de Física Fonamental, Universitat de Barcelona, Carrer Martí i Franqués 1, 08028-Barcelona, Spain

PACS 46.50.+a - Fracture mechanics, fatigue and cracks

PACS 62.20.Mk - Fatigue, brittleness, fracture, and cracks

PACS 64.60.Ak - Renormalization-group, fractal, and percolation studies of phase transitions

\begin{abstract}
We study the effect of strong heterogeneities on the fracture of disordered materials using a fiber bundle model. The bundle is composed of two subsets of fibers, i.e. a fraction $0 \leq \alpha \leq 1$ of fibers is unbreakable, while the remaining $1-\alpha$ fraction is characterized by a distribution of breaking thresholds. Assuming global load sharing, we show analytically that there exists a critical fraction of the components $\alpha_{c}$ which separates two qualitatively different regimes of the system: below $\alpha_{c}$ the burst size distribution is a power law with the usual exponent $\tau=5 / 2$, while above $\alpha_{c}$ the exponent switches to a lower value $\tau=9 / 4$ and a cutoff function occurs with a diverging characteristic size. Analyzing the macroscopic response of the system we demonstrate that the transition is conditioned to disorder distributions where the constitutive curve has a single maximum and an inflexion point defining a novel universality class of breakdown phenomena.
\end{abstract}

Introduction. - Damage and fracture of materials occurring under various types of external loads is a very important scientific problem with an enormous technological impact. During the last two decades the application of statistical physics has revealed that heterogeneities of materials' microstructure play a crucial role in fracture prociesses [1]. To capture the effect of disorder, recently several stochastic fracture models have been proposed such às the fiber bundle model (FBM) and lattice models of fuses or springs [1-7]. Based on these models, analytic calc culations and computer simulations revealed that macroscopic fracture of disordered materials shows interesting annalogies with phase transitions and critical phenomena having several universal features independent of specific material details $[1,4-6,8-10]$. It has been found that under a slowly increasing external load macroscopic failure is preceded by a bursting activity due to the cascading nature of local breakings $[3,4]$. Since the bursts can be recorded experimentally by the acoustic emission technique, these precursors addressed the possibility of forecasting the imminent failure event [11-14]. The size distribution of bursts was proven to be a power law with an exponent which is universal for a broad class of disorder distributions [3,4]. Recently, the robustness of the universality class has been tested by mixing different types of disorder distributions [17], and by introducing a gap into the domain of strength values [18]. However, relevant change of the burst size distribution was only obtained when introducing a finite lower threshold for the strength disorder. Increasing the threshold strength a crossover occurs from a power law of exponent $5 / 2$ to another one with a lower exponent $3 / 2$ [14-16]. Divakaran and Dutta have studied the critical behaviour of a Random Fiber Bundle Model with mixed uniform distribution of threshold strengths [18]. They have considered two uniform distributions separated by a gap. The approach developed in this Letter might be interpreted as the infinite gap limit of Divakaran's model.

In the present paper we study the effect of strong heterogeneities on the process of fracture based on a fiber bundle model. We assume that the system has two components one of which is characterized by a strength distribution, while the other one is unbreakable. Varying the fraction of the two components $\alpha$ under global load sharing conditions, we show analytically that the presence of unbreakable elements has a substantial effect on the fracture process of the system both on the micro- and macroscales. Very interestingly, we find a critical fraction $\alpha_{c}$ where a transition occurs between two qualitatively different regimes: below the critical point $\alpha<\alpha_{c}$ the macroscopic constitutive curve has a single maximum and the burst size distribution is a power law with the usual mean field exponent $\tau=5 / 2$. However, above $\alpha_{c}$ the macroscopic response becomes monotonous and the burst exponent switches to a lower value $\tau=9 / 4$ with a cutoff function. Based on the analysis of the macroscopic response of the system, we show that the transition is conditioned to disorder distributions where the constitutive curve has 
a single maximum and an inflection point defining a novel universality class of breakdown phenomena.

Model. - We consider a set of $N$ fibers which are loaded in parallel. Under an increasing external load $\sigma_{o}$ the fibers have a linearly elastic response with a Young modulus $E=1$ fixed for all the fibers. In order to capture the large variation of disordered material properties, we assume that the bundle is composed of two subsets of fibers with strongly different breaking characteristics: A fraction $0 \leq \alpha \leq 1$ of fibers is strong in the sense that they have an infinite load bearing capacity so that they never break. However, fibers of the remaining $1-\alpha$ fraction are weak and break when the load on them $\sigma$ exceeds a threshold value $\sigma_{t h}^{i}, i=1, \ldots, N_{w}$, where $N_{w}=(1-\alpha) N$ is the number of weak fibers. The strength disorder of weak fibers is characterized by the probability density $p\left(\sigma_{t h}\right)$ and distribution function $P\left(\sigma_{t h}\right)=\int_{0}^{\sigma_{t h}} p(x) d x$ of the failure thresholds. After a weak fiber breaks in the bundle, its load has to be overtaken by the remaining intact ones. For simplicity, we assume global load sharing (GLS) (also called equal load sharing) which means that all the intact fibers share the same load $\sigma$, hence, no stress concentration occurs around failed regions. Under these conditions the constitutive equation of the model can be written as

$$
\sigma_{o}=(1-\alpha)[1-P(\sigma)] \sigma+\alpha \sigma,
$$

where $\sigma_{o}$ is the external load acting on the sample and $\sigma$ denotes the load of single fibers which is related to the strain $\varepsilon$ of the system as $\sigma=E \varepsilon$. The first term of Eq. (11) accounts for the load bearing capacity of the surviving fraction of weak elements, and the second one represents the stress carried by the unbreakable subset of the system. In the following calculations it is instructive to consider two different strength distributions for the weak fibers, namely, a uniform distribution between 0 and 1 and a Weibull distribution will be used with the distribution functions $P(\sigma)=\sigma$ and $P(\sigma)=1-\exp \left[-(\sigma / \lambda)^{m}\right]$, respectively.

The constitutive behavior of the system is presented in Fig. 1, for the two different disorder distributions. We recover the usual FBM solutions [2] in the limiting case of $\alpha=0$, when the bundle is only composed of weak fibers. Those solutions usually present a parabolic maximum, which defines the critical deformation $\sigma_{c}=E \varepsilon_{c}$ and critical strength $\sigma_{o}^{c}\left(\sigma_{c}\right)$ of the system. For finite values of $\alpha$, all the weak fibers break for large enough $\sigma$ so that the first term of Eq. (11) goes to zero while the unbreakable fibers overtake the entire external load. Consequently, the constitutive curves in Fig. 1 tend asymptotically to a straight line with slope $\alpha E$. It can be seen in Fig. 1 that for low values of $\alpha$ the local maximum of $\sigma_{o}(\sigma)$ prevails but its position $\sigma_{c}(\alpha)$ and value $\sigma_{o}^{c}(\alpha)$ are monotonically increasing with $\alpha$. It is interesting to note that there exists a well defined critical value of the fraction of the components $\alpha_{c}$ above which $\alpha>\alpha_{c}$ the local maximum disappears and the constitutive curve becomes a monotonically increasing
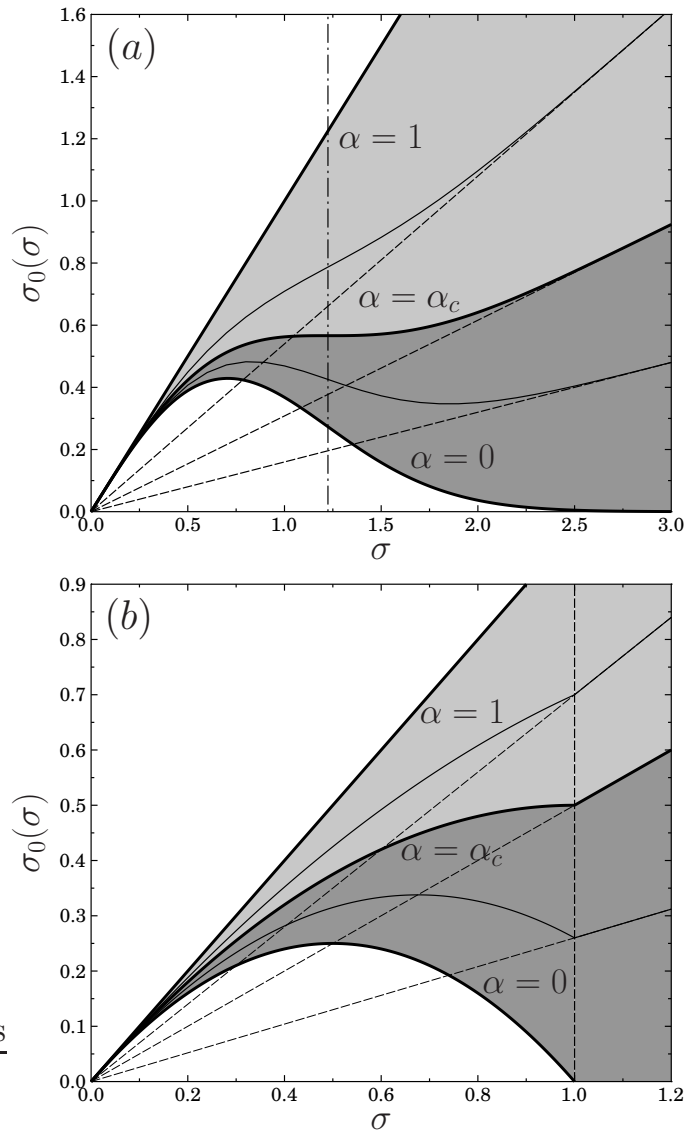

Fig. 1: Constitutive behavior of the system for several values of $\alpha$ using a Weibull distribution with $m=2$ and $\lambda=1(a)$, and uniformly distributed threshold values $(b)$. The two regimes $\alpha<\alpha_{c}$ and $\alpha>\alpha_{c}$ are indicated by the grey areas, and the dashed lines with slope $\alpha$ show the asymptotic linear behavior of $\sigma_{o}(\sigma)$. In the figures, the vertical straight lines represent the position of the inflexion point and the value of the largest breaking threshold for the Weibull $(a)$ and uniform distributions (b), respectively.

function $d \sigma_{o} / d \sigma>0$. The position of the maximum $\sigma_{c}$ is obtained from the condition of extreme $\left.\frac{d \sigma_{o}}{d \sigma}\right|_{\sigma_{c}}=0$, which can be cast into the form

$$
\frac{1}{1-\alpha}=P\left(\sigma_{c}\right)+\sigma_{c} p\left(\sigma_{c}\right)
$$

The above equation should be solved for $\sigma_{c}$ as a function of $\alpha$, then $\sigma_{o}^{c}$ can be determined by substituting $\sigma_{c}(\alpha)$ into Eq. (11). Since the derivative of the constitutive curve $\sigma_{o}(\sigma)$ has a minimum in the inflexion point $\sigma_{i n}$, the right hand side of Eq. (2) has a maximum at $\sigma_{c}=\sigma_{i n}$. It follows that Eq. (2) can only be solved for $\sigma_{c}(\alpha)$ until $\alpha \leq \alpha_{c}$, where the critical fraction of strong fibers $\alpha_{c}$ is defined as the solution of $\sigma_{c}\left(\alpha_{c}\right)=\sigma_{i n}$. It is important to emphasize that the location of the inflexion point $\sigma_{i n}$ does not depend on the value of $\alpha$ since the second derivative 
of the constitutive equation $\sigma_{o}(\sigma)$ reads as

$$
\left.\frac{d^{2} \sigma_{o}}{d^{2} \sigma}\right|_{\sigma_{c}}=-(1-\alpha)\left[2 p(\sigma)+\sigma p^{\prime}(\sigma)\right] .
$$

For the case of Weibull distributions the general solution $\sigma_{c}(\alpha)$ cannot be obtained in a closed form. However, one can still calculate analytically $\alpha_{c}$ and determine $\sigma_{c}$ for two parameter values $\alpha=0$ and $\alpha=\alpha_{c}$. The calculations result in $\sigma_{c}(\alpha=0)=\lambda(1 / m)^{1 / m}$, and $\sigma_{c}\left(\alpha_{c}\right)=\sigma_{i n}$, where the inflexion point is $\sigma_{i n}=\lambda[(1+m) / m]^{1 / m}$. The critical point $\alpha_{c}$ is then obtained by substituting $\sigma_{i n}$ into Eq. (2) which yields $\alpha_{c}=m e^{-(1+m) / m} /\left[1+m e^{-(1+m) / m}\right]$.

Note that the above arguments do not apply to the uniform distribution, since the constitutive curve $\sigma_{o}=$ $[1-(1-\alpha) \sigma] \sigma$ does not have an inflexion point (see Fig. $1(b)$ ). The position of the maximum of $\sigma_{o}(\sigma)$ can be obtained analytically as $\sigma_{c}(\alpha)=1 / 2(1-\alpha)$, which holds for $\alpha \leq \alpha_{c}$ with the critical value of the control parameter $\alpha_{c}=1 / 2$. At $\alpha_{c}$ the value of $\sigma_{c}$ coincides with the upper bound of strength values $\sigma_{t h}^{\max }=1$. The parabolic shape of the constitutive curve prevails even for $\alpha>\alpha_{c}$ but $\sigma_{o}(\sigma)$ becomes linear at $\sigma=\sigma_{t h}^{\max }$ before reaching the maximum, so that the rest of the parabola cannot be realized.

The presence of the critical point and the qualitatively different forms of $\sigma_{o}(\sigma)$ below and above $\alpha_{c}$ have a substantial effect on the microscopic breaking of the system. Under stress controlled loading conditions the decreasing part of $\sigma_{o}(\sigma)$ can not be accessed for $\alpha<\alpha_{c}$. Contrary, a horizontal jump occurs giving rise to a large number of breakings in one step. For uniformly distributed failure thresholds, this unstable avalanche is the last one that includes all the remaining weak fibers. In the Weibull case, however, the threshold values are distributed over an infinite domain, so that the jump is still followed by breaking events which disappear only asymptotically. For the detailed characterization of the microscopic breaking process, we analyze the size distribution of bursts of fiber breakings.

Precursory activity. - Under stress controlled loading conditions, each fiber breaking is followed by the redistribution of load over the intact elements. Assuming global load sharing the load is everywhere the same $\sigma$ in the system. When the external load is increased quasistatically, i.e. $\sigma_{o}$ is increased to break only a single fiber, the subsequent load redistribution triggers an entire burst of breakings. In the simple FBM these local failure events result in fluctuating burst sizes $\Delta$, with an increasing average, as macroscopic failure is approached. The size distribution of the bursts is one of the most important characteristics of the microscopic fracture process which can be monitored experimentally by the acoustic emission techniques. It has been demonstrated that in FBM under GLS conditions [4], the burst size distribution can be obtained analytically in the form of an integral

$$
\frac{D(\Delta)}{N}=\frac{\Delta^{\Delta-1}}{\Delta !} \int_{0}^{\sigma_{m}} p(\sigma)\left(1-a_{\sigma}\right) a_{\sigma}^{\Delta-1} e^{-a_{\sigma} \Delta} d \sigma,
$$

where $a_{\sigma}=\sigma p(\sigma) /[1-P(\sigma)]$ is the average number of fibers which break as a consequence of a single fiber failure at the load $\sigma$. It was shown in Refs. $[4,14,15]$ that the distribution $D(\Delta)$ simplifies to a power law $D(\Delta) \sim \Delta^{-\tau}$ with the exponent $\tau=5 / 2$ for a broad class of disorder distributions where the constitutive curve of the system has a single quadratic maximum.

In the following we show analytically that in the presence of unbreakable fibers, the avalanche statistics changes and a novel universality class of FBMs emerges. Slowly increasing the external load to break a single fiber, its failure stress $\sigma$ is equally redistributed over the intact fibers giving rise to the load increment

$$
\delta \sigma=\frac{\sigma}{N[1-P(\sigma)](1-\alpha)+\alpha N} .
$$

It can be seen that the strong fibers reduce the load increment $\delta \sigma$ on the weak ones, since the load beared by the strong fibers does not contribute to breaking. The average number of fibers $a_{\sigma}$ which fail as a consequence of this increment $\delta \sigma$ can be cast into the form

$$
a_{\sigma}=(1-\alpha) N p(\sigma) \delta \sigma=\frac{(1-\alpha) \sigma p(\sigma)}{\alpha+(1-\alpha)[1-P(\sigma)]} .
$$

The size distribution of the resulting bursts can be obtained by substituting Eq. (6) into the general expression Eq. (4), where we have to analyze the behavior of the integral

$$
I(\Delta) \equiv \int_{0}^{\sigma_{m}} p(\sigma) \frac{1-a_{\sigma}}{a_{\sigma}} e^{-\Delta\left[a_{\sigma}-\ln a_{\sigma}\right]} d \sigma,
$$

for different values of $\alpha$. The upper integral limit corresponds to the location of the maximum in the constitutive curve $\sigma_{m}$. For large $\Delta$ this integral is controlled by the maximum of the exponent. The extreme condition of $\psi \equiv a_{\sigma}-\ln a_{\sigma}$ result in $\psi^{\prime}=a_{\sigma}^{\prime}\left(1-\frac{1}{a_{\sigma}}\right)=0$, corresponding to a maximum at $a_{\sigma}=1$. Below the critical point $\alpha<\alpha_{c}$, for $a_{\sigma}<1$, we can make the expansions

$$
a_{\sigma} \simeq 1+\left.a_{\sigma}^{\prime}\right|_{\sigma_{m}}\left(\sigma-\sigma_{m}\right),
$$

and

$$
\psi \simeq 1+\left.\frac{a_{\sigma}^{\prime 2}}{2}\right|_{\sigma_{m}}\left(\sigma-\sigma_{m}\right)^{2} .
$$

Inserting Eqs. (819) into the expression of $I(\Delta)$ we get

$$
I(\Delta) \simeq p\left(\sigma_{m}\right) a_{\sigma}^{\prime} e^{-\Delta} \int_{0}^{\sigma_{m}}\left(\sigma-\sigma_{m}\right) e^{-\frac{\Delta a_{\sigma}^{\prime}}{2}\left(\sigma-\sigma_{m}\right)^{2}} d \sigma .
$$

Substituting $I(\Delta)$ into the general equation Eq. (4) and taking the large $\Delta$ limit of the prefactor, the asymptotic 
behavior of the burst size distribution can be cast in the form

$$
\frac{D(\Delta)}{N} \simeq \frac{p\left(\sigma_{m}\right)}{\sqrt{2 \pi} a_{\sigma}^{\prime}} \Delta^{-5 / 2}
$$

which coincides with the known result of Refs. $[4,14,15]$ in the limit $\alpha=0$. This derivation implies that the presence of a finite amount of unbreakable fibers does not change qualitatively the behavior of the system while the single quadratic maximum of the constitutive curve prevails $\alpha<$ $\alpha_{c}$.

The situation drastically changes when we reach $\alpha_{c}$, since at this point the position of the maximum $\sigma_{c}\left(\alpha_{c}\right)$ and of the inflexion point $\sigma_{i n}$ of the constitutive curve coincide with each other so that $\left.\frac{d \sigma_{o}}{d \sigma}\right|_{\sigma_{c}}=0$ and $\left.\frac{d^{2} \sigma_{o}}{d \sigma^{2}}\right|_{\sigma_{c}}=0$ hold. Above $\alpha_{c}$ no maximum of the constitutive curve exists $d \sigma_{o} / d \sigma>0$. It can easily be shown that at $\alpha=\alpha_{c}$ the average number of failing fibers $a_{\sigma}$ as a consequence of a single fiber breaking has the properties $a_{\sigma_{c}}=1$ and $\left.a_{\sigma}^{\prime}\right|_{\sigma_{c}}=0$. In order to determine the asymptotic behavior of $I(\Delta)$ it is then necessary to carry out the Taylor expansions of Eqs. (819) to the next order. In this case we obtain for $a_{\sigma}$ and $\psi$

$$
a_{\sigma} \simeq 1+\frac{a^{\prime \prime}{ }_{\sigma}}{2}\left(\sigma-\sigma_{c}\right)^{2}, \quad \psi \simeq 1+\frac{3{a_{\sigma}}^{\prime \prime 2}}{4 !}\left(\sigma-\sigma_{c}\right)^{4} .
$$

Inserting these expressions into Eq. (7), the integral can be cast into the form

$$
I(\Delta) \simeq \frac{p\left(\sigma_{c}\right) a_{\sigma}^{\prime \prime} e^{-\Delta}}{2} \int_{0}^{\infty} d \sigma\left(\sigma-\sigma_{c}\right)^{2} e^{\frac{3 a^{\prime \prime 2}{ }_{\sigma} \Delta}{4 !}\left(\sigma-\sigma_{c}\right)^{4}} .
$$

Following the usual procedure, we arrive at

$$
I(\Delta) \simeq \frac{\Gamma\left(\frac{3}{4}\right)}{2^{5 / 2} 3^{7 / 4} a_{\sigma}^{\prime \prime 1 / 2}} e^{-\Delta} \Delta^{-3 / 4}
$$

which implies that at the critical point $\alpha_{c}$ the avalanche size distribution changes to

$$
\frac{D(\Delta)}{N} \simeq \frac{\Gamma\left(\frac{3}{4}\right)}{24 \sqrt{3 \pi a_{\sigma}^{\prime \prime}} 3^{1 / 4}} \Delta^{-9 / 4}
$$

Our derivation demonstrates that increasing $\alpha$ the behavior of the system changes both on the macro- and the micro-scales. We showed that while the quadratic maximum of $\sigma_{o}(\sigma)$ prevails, i.e. below the critical point $\alpha_{c}$, the asymptotic behavior of the burst size distribution $D(\Delta)$ is controlled by the vicinity of the maximum resulting in a power law functional form $D(\Delta) \sim \Delta^{-\tau}$ with an universal exponent $\tau=5 / 2$. However, at $\alpha_{c}$ the constitutive curve becomes monotonically increasing $d \sigma_{o} / d \sigma>0$ and the avalanche statistics is dominated by the inflexion point of $\sigma_{o}(\sigma)$, giving rise to a different value of the exponent $\tau=9 / 4$. Varying the control parameter $\alpha$, the exponent $\tau$ suddenly switches between the two values $5 / 2$ and $9 / 4$ when passing the critical point $\alpha_{c}$. Note that in the derivation the only assumption we made is that the constitutive
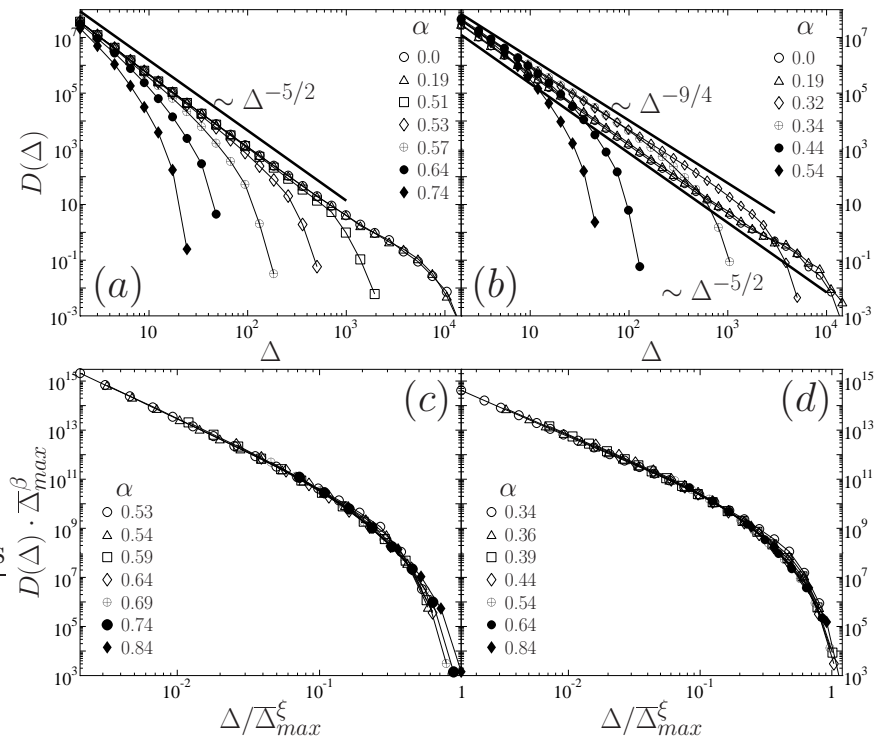

Fig. 2: Non-normalized avalanche size distributions for uniform $(a, c)$ and Weibull distributions $(b, d)$ varying $\alpha$ below and above $\alpha_{c}$. The straight lines in $(a)$ and $(b)$ represent the power laws obtained analytically Eqs. (1115). Rescaling the two axis according to the scaling formula Eq. (16), a very good quality data collapse is obtained in $(c)$ and $(d)$.

curve of the system has a single maximum and an inflexion point. It follows that the change of the exponent $\tau$ of the avalanche size distribution can be observed for a large variety of disorder distributions defining a novel universality class of breakdown phenomena. This universality class is narrower than the one in which the power law behavior of $D(\Delta)$ emerges with the exponent $\tau=5 / 2$. For instance, the Weibull distributions do present the above switching of exponents, however, the uniform distribution does not.

We have carried out Monte Carlo simulations to validate the previous theoretical predictions. We have explored the quasi-static fracture process of our fiber bundle model using computer simulations of a system composed of $N=$ $10^{6}$ fibers and averaging over $10^{3}$ samples both for uniform and Weibull distributions for the fiber breaking thresholds.

Figure 2 displays the burst statistics for a fiber bundle with uniform and Weibull failure thresholds in Figs. $2(a, c)$ and Figs. 2(b, $d)$, respectively. In both cases, below the critical fraction of unbreakable fibers, $\alpha_{c}$, the burst distributions $D(\Delta)$ do not change significantly, and even the cutoffs associated to the lack of numerical statistics do not change with $\alpha$. For a uniform distribution of breakable fibers, the constitutive equation for $\sigma \leq \sigma_{c}(\alpha)$ reduces to the one corresponding to a material composed only by weak fibers, with threshold values between zero and the upper bound $\sigma_{t h}^{\max }=1 /(1-\alpha)$. Hence, it follows that the entire failure process of the bundle, obtained at different $\alpha$ values, remains the same until there are enough weak fibers in the system $N_{w}>N / 2$. Furthermore, for the parameter regime $\alpha<\alpha_{c}$ the avalanche statistics does 

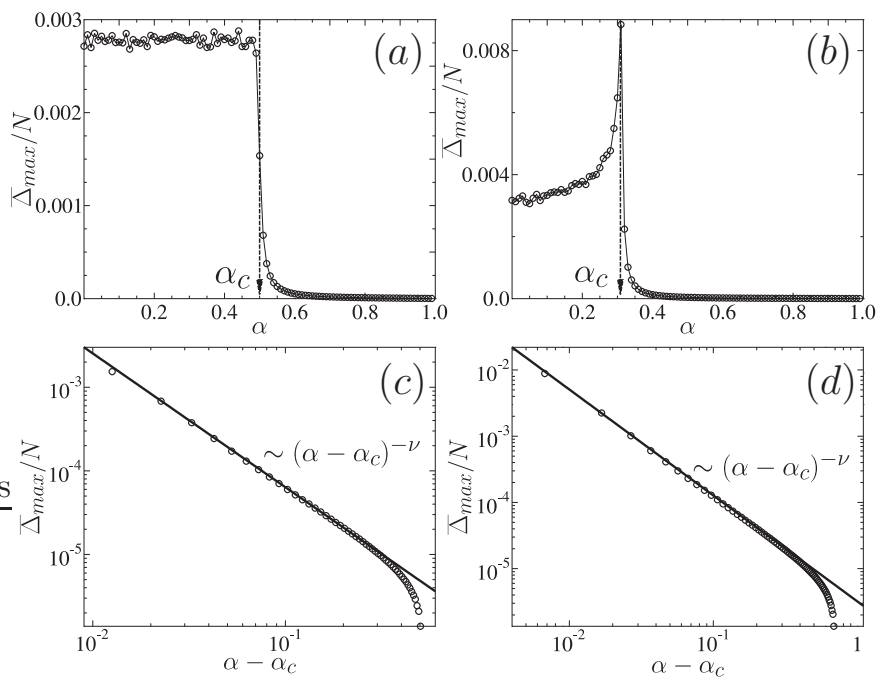

Fig. 3: The average size of the largest burst $\bar{\Delta}_{\max }$ as a function of $\alpha$ for uniform $(a, c)$ and Weibull $(b, d)$ distributions. The vertical straight lines in the figures indicate the corresponding critical point $\alpha_{c}$. $(c)$ and $(d)$ show that approaching $\alpha_{c}$ from above, $\bar{\Delta}_{\max }$ has a power law divergence as a function of $\alpha-\alpha_{c}$. The value of the exponent is $\nu=1.56 \pm 0.07$ for both cases.

not change. We obtain the typical power law distribution $D(\Delta) \sim \Delta^{-\tau}$ with the exponent $\tau=5 / 2$ (Figs. $2(a, c)$ ). On the other hand, the parabolic shape of the constitutive behavior $\sigma_{o}(\sigma)$ also prevails for $\alpha>\alpha_{c}$. In this regime, the system behaves as if the loading process was stopped before reaching the maximum of $\sigma_{o}(\sigma)$, due to the insufficient number of breakable fibers $N_{w}<N / 2$ (compare to Fig. 1 $(a)$ ). Consequently, the cutoff of the distribution $D(\Delta)$ in Fig. 2 $(a)$ decreases with increasing $\alpha$. However, the exponent $\tau$ keeps the same value as below $\alpha_{c}$, in agreement with our predictions and with Ref. [4].

We use the average size of the largest burst $\bar{\Delta}_{\max }$ as the characteristic burst size of the system. It can be seen in Fig. 3( $(a)$ that for the uniform distribution below $\alpha_{c}$, the value of $\bar{\Delta}_{\max }$ is constant, while it decreases rapidly when $\alpha$ surpasses $\alpha_{c}$. Figure $3(c)$ demonstrates that approaching $\alpha_{c}$ from above the characteristic burst size shows a power law divergence $\bar{\Delta}_{\max } \sim\left(\alpha-\alpha_{c}\right)^{-\nu}$. The value of the exponent $\nu=1.56 \pm 0.07$ was obtained numerically.

In Figures $2(b, d)$, the burst statistics of a fiber bundle with a Weibull distribution of breaking thresholds is illustrated. The Weibull parameters were set to $m=2$ and $\lambda=1$, corresponding to a critical point $\alpha_{c} \simeq 0.3085$. In this case, below the critical point $\alpha<\alpha_{c}$ the burst size distribution has a power law behavior $D(\Delta) \sim \Delta^{-\tau}$, with the exponent $\tau=5 / 2$ (Fig. 2(b)). In this regime, a slight increase of the cutoff burst size appears when increasing $\alpha$, however, the power law part of the distribution does not change. When $\alpha$ surpasses $\alpha_{c}$, the exponent of the power law regime of $D(\Delta)$ suddenly switches to the lower value $\tau=9 / 4$. The latter is in excellent agreement with our analytic predictions (see Fig. 2(b)). Moreover, we again find that the characteristic burst size $\bar{\Delta}_{\max }$ diverges as a power law as we approach $\alpha_{c}$ from above. The value of the critical exponent $\nu$ is the same as for the uniform case (see Fig. 3 $(d)$ ). We emphasize that the value of the exponent of the power law regime of $D(\Delta)$ remains constant $\tau=9 / 4$ when changing $\alpha$ above $\alpha_{c}$.

Using $\bar{\Delta}_{\max }$ as a scaling variable, we introduce the scaling ansatz

$$
D(\Delta)=\bar{\Delta}_{\max }^{-\beta} g\left(\Delta / \bar{\Delta}_{\max }^{\xi}\right)
$$

for the burst size distributions above the critical point $\alpha>$ $\alpha_{c}$. Here $\beta$ and $\xi$ are scaling exponents, which have the relation $\beta=\tau \xi$ with $\tau=5 / 2$ and $\tau=9 / 4$ for the uniform and Weibull distributions, respectively. Figures $2(c)$ and (d) present the rescaled burst size distributions plotting $D(\Delta) \bar{\Delta}_{\max }^{\beta}$ as a function of $\Delta / \bar{\Delta}_{\max }^{\xi}$. The high quality data collapse is obtained with the parameters $\beta=3.25$, $\xi=1.25$ and $\beta=2.52, \xi=1.12$, for the uniform and Weibull distributions, which are consistent with the two different values of the $\tau$ exponent.

Discussion. - Our numerical and analytical calculations revealed that the presence of unbreakable elements gives rise to a substantial change of the fracture process of disordered materials both on the micro- and macro-scales. Astonishingly we found a critical fraction of the breakable and unbreakable components where the exponent of the burst size distribution switches from the well known mean field exponent of FBM $\tau=5 / 2$ to a significantly lower value $\tau=9 / 4$. The transition is conditioned to disorder distributions where the macroscopic constitutive response of the system has a single maximum and an inflexion point, implying a novel universality class of FBM. Despite we have considered only unbreakable fibers, our results will hold for a finite gap of two threshold distributions down to a certain critical value of the gap size. Below this critical value our model recovers the former work of Ref. [18]. Besides its theoretical importance, the problem has several implications for experimental studies. New materials of high mechanical performance are often fabricated by mixing components with widely different properties. For instance, fiber reinforced composites are composed of strong fibers which are embedded in a carrier matrix. In this case at the breaking of weak elements, the strong ones act as the unbreakable component of our model.

Our detailed analytical and numerical study is restricted to the quasi-static limit of FBMs where the external load is incremented in a continuous manner. However, in laboratory experiments only finite discrete load steps can be realized. Recently, it has been demonstrated that for large enough load increments the statistics of bursts changes, i.e. under GLS conditions the exponent of the power law distribution of burst sizes takes a higher value $\tau=3.0[19,20]$. When the system is a mixture of weak and strong fibers the effect of finite load steps depends on the type of dis- 
order. For uniformly distributed failure thresholds it is straightforward to show that the burst exponent changes to $\tau=3.0$ both below and above the critical fraction $\alpha_{c}$. Nevertheless, disorder distributions for which the constitutive curve has an inflexion point (e.g. Weibull distribution) deserve a detailed study which will be presented elsewhere.

A very interesting application of FBMs is to study the time dependent deformation and rupture of disordered materials under a constant external load (creep rupture). To understand damage enhanced creep processes the relaxation dynamics of FBMs has recently been investigated in details [21-23]. It has been found that below the critical load $\sigma_{c}$ the system suffers only partial failure and relaxes to a stable state, while above $\sigma_{c}$ macroscopic breaking occurs in a finite time. When approaching $\sigma_{c}$ from either side, the characteristic time scale (relaxation time and lifetime of the system) has a power law divergence with a universal exponent $1 / 2[22,23]$. Since the relaxation dynamics of the system is determined by the functional form of the constitutive curve in the vicinity of the critical load, novel behaviour can be expected in the presence of strong fibers. The relaxation dynamics and creep rupture open up interesting possibilities for future applications of our model.

Acknowledgments. - This work is part of the Spanish-Hungarian Intergovernmental Scientific Project HH2005-0016. RCH acknowledges the financial support of the Spanish Minister of Education and Science, through a Ramon y Cajal Program. IP thanks Spanish M.E.C. (FIS2005-01299) and DURSI (Distinción de la Generalitat de Catalunya) (Spain) for financial support. KK and FK were also supported by OTKA T049209 and NKFP$3 \mathrm{~A} / 043 / 04$.

\section{REFERENCES}

[1] Alava M, Nukala P.K.V., and Zapperi S., Advances in Physics, 55 (2006) 349

[2] Zapperi A, Vespignani A and Stanley HE, Nature, 388 (1997) 658

[3] Hansen A. and Hemmer P. C., Phys. Lett. A, 184 (1994) 394

[4] Kloster M., Hansen A., and Hemmer P. C., Phys. Rev. E, 56 (1997) 2615

[5] Kun F., Zapperi S. and Herrmann H.J., Eur. Phys. J. B, 17 (2000) 269

[6] Hidalgo R.C., Moreno Y., Kun F. and Herrmann H.J., Phys. Rev. E, 65 (2002) 046148

[7] Hidalgo R.C., Grosse C.U., Kun F., Reinhardt H.W. and Herrmann H.J., Phys. Rev. Lett., 89 (2002) 025501

[8] Moreno Y., Gomez J. B. and Pacheco A. F., Phys. Rev. Lett., 85 (2000) 2865

[9] Pradhan S., Chakrabarti B.K. and Hansen A., Phys. Rev. E, 71 (2005) 036149

[10] Raischel F., Kun F. and Herrmann H.J., Phys. Rev. E, 73 (2006) 066101
[11] Petri A., Paparo G., Vespignani A., Alippi A. and Costantini M.,, Phys. Rev. Lett., 73 (1994) 3423

[12] Garcimartin A., Guarino A., Bellon L. and CilibERTo S., Phys. Rev. Lett., 79 (1997) 3202

[13] Pradhan S. and Chakrabarti B. K., Phys. Rev. E, 65 (2002) 016113

[14] Pradhan S., Hansen A. and Hemmer P. C., Phys. Rev. Lett., 95 (2005) 125501

[15] Pradhan S., Hansen A. and Hemmer P. C., Phys. Rev. E, 74 (2006) 016122

[16] Pradhan S. and Hansen A., Phys. Rev. E, 72 (2005) 026111

[17] Divakaran U. and Dutta A., Phys. Rev. E, 75 (2007) 011109

[18] Divakaran U. and Dutta A., Phys. Rev. E, 75 (2007) 011117

[19] Pradhan S., Bhattacharyya P., and Chakrabarti B. K., Phys. Rev. E, 66 (2002) 016116

[20] Hemmer P. C. and Pradhan S., Phys. Rev. E, 75 (2007) 046101

[21] Kun F., Moreno Y., Hidalgo R. C., and Herrmann H. J., Europhys. Lett., 63 (2003) 347

[22] Kun F., Hidalgo R. C., Herrmann H. J., and Pál K. F., Phys. Rev. E, 67 (2003) 061802

[23] Pradhan S. and Hemmer P. C., Phys. Rev. E, 75 (2007) 056112 